\title{
The WEBT campaigns on BL Lacertae
}

\section{Time and cross-correlation analysis of optical and radio light curves 1968-2003*}

\author{
M. Villata ${ }^{1}$, C. M. Raiteri ${ }^{1}$, H. D. Aller ${ }^{2}$, M. F. Aller ${ }^{2}$, H. Teräsranta $^{3}$, P. Koivula ${ }^{3}$, S. Wiren ${ }^{3}$, \\ O. M. Kurtanidze ${ }^{4,5,6}$, M. G. Nikolashvili ${ }^{4}$, M. A. Ibrahimov ${ }^{7}$, I. E. Papadakis ${ }^{8,9}$, G. Tosti ${ }^{10}$, \\ F. Hroch ${ }^{11}$, L. O. Takalo ${ }^{12}$, A. Sillanpää ${ }^{12}$, V. A. Hagen-Thorn ${ }^{13,14}$, V. M. Larionov ${ }^{13,14}$, \\ R. D. Schwartz ${ }^{15}$, J. Basler ${ }^{15}$, L. F. Brown ${ }^{16}$, and T. J. Balonek ${ }^{17}$
}

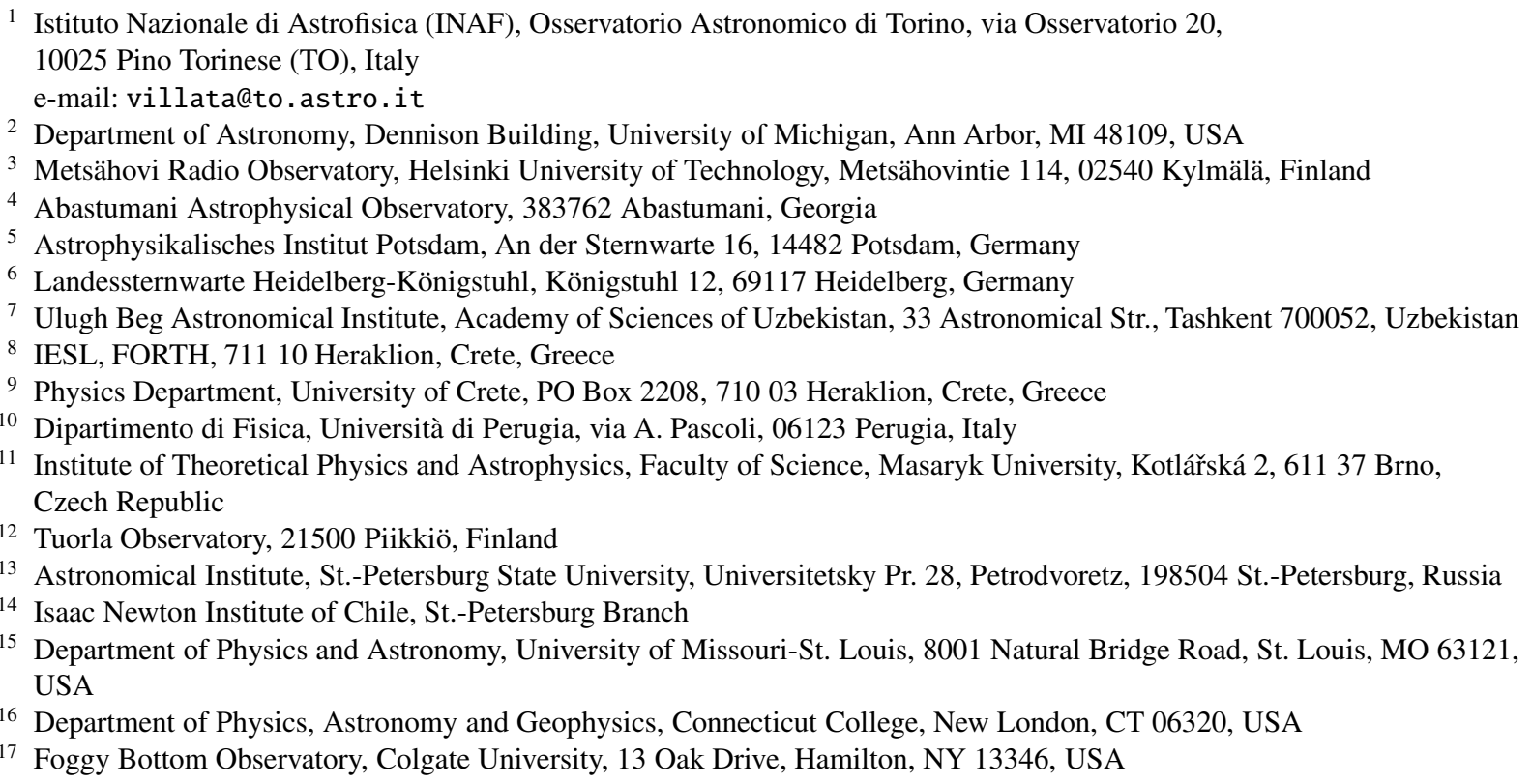

Received 15 March 2004 / Accepted 27 May 2004

\begin{abstract}
The Whole Earth Blazar Telescope (WEBT) collaboration has collected a large amount of optical and radio data on BL Lacertae in the last years, which, when added to literature data, allow to construct well-sampled light curves of the source from 1968 to the end of 2003. These optical and radio data are here analysed with three statistical methods designed for unevenly-sampled data trains in order to search for possible periodicities. While the main radio outbursts repeat every $\sim 8$ years, with a possible progressive stretching of the period, the evidence of an optical periodicity is much less clear. Radio light curves from 4.8 to $37 \mathrm{GHz}$ are well correlated, with variations at the higher frequencies leading the lower-frequency ones by a few weeks for contiguous bands, up to a few months when considering the largest frequency separations. The radio behaviour reveals the presence of two different components, the softer-spectrum one constituting the bulk of the radio emission. On the other hand, the harder component shows itself as radio events which appear enhanced at the higher frequencies and seem to have optical counterparts. Cross-correlation between the optical light curve and radio hardness ratios indicates a radio time delay of more than 3 months. Thus, our analysis suggests a scenario where flux variations propagate towards less and less opaque regions, giving rise to related optical and hard radio events and, in more extended zones, to soft events apparently uncorrelated with the former ones.
\end{abstract}

Key words. galaxies: BL Lacertae objects: general - galaxies: BL Lacertae objects: individual: BL Lacertae - galaxies: jets galaxies: quasars: general

^ For questions regarding the availability of the data discussed in this paper, please contact Massimo Villata (villata@to.astro.it).

\section{Introduction}

BL Lacertae is the prototype of a class of active galactic nuclei, the BL Lac objects, which are well known for their 
pronounced variability at all wavelengths, from the radio to the $\gamma$-ray band. The commonly accepted scenario that best explains most of the observations foresees a black hole ejecting a nonthermally emitting plasma jet which points close to the line of sight. Although the general framework seems to be well understood, the variation mechanisms appear to be less constrained from an observing point of view and different phenomena are invoked, such as shock waves travelling downstream, magnetic reconnection events, plasma blobs ejected from the core and possibly following complex geometric paths, variations of the Doppler factor of geometrical nature (e.g., Villata \& Raiteri 1999). In order to tackle this issue, a huge observational work is needed to monitor the sources as densely as possible, and this can only be achieved through the joint effort of many observers.

The international collaboration among optical and radio astronomers known as the Whole Earth Blazar Telescope (WEBT; http://www . to.astro.it/blazars/webt/; e.g., Villata et al. 2000, 2002) was born in 1997 to reach this goal. A dozen campaigns have been organized up to now on several BL Lac objects. In particular, four campaigns targeted BL Lacertae: two of them in 1999 were short-term campaigns run in conjunction with pointings by the X-ray satellites ASCA and BeppoSAX (Ravasio et al. 2002), a long-term campaign was carried out in May 2000-January 2001 (BL Lac 2000; Villata et al. 2002; see also Böttcher et al. 2003), and another long-term campaign in May 2001-February 2002 (BL Lac 2001). Then, when collecting data from this last campaign, it was decided to reconstruct also the "historic" light curves of this object. This led to a more ambitious project, which was called "BL Lac 2001 and its extension". In a previous paper (Villata et al. 2004) optical light curves from 1994 to 2002 were presented and a detailed colour analysis was performed. In the present paper optical and radio data back to 1968 are considered and searched for periodicities and correlations by means of statistical tools specifically designed to analyse unevenlysampled time series.

The existence of a periodic or quasi-periodic behaviour in the optical and/or radio light curves of some BL Lac objects has been recognized since many years (see e.g. the cases of OJ 287 and AO 0235+16 in Sillanpää et al. 1996, and Raiteri et al. 2001, respectively), and several theoretical models to interpret periodicity have been proposed, mainly dealing with binary black holes scenarios (e.g., Villata et al. 1998; Ostorero et al. 2004, and references therein). In particular, the radio and optical light curves of BL Lacertae have been investigated for periodicities in a number of works, with different results. The Fourier transform method was used by Carini et al. (1992) to analyse 10 years of optical monitoring of the source, finding no clear evidence of a periodicity. Smith \& Nair (1995) applied visual inspection, structure function, and power spectrum techniques to the optical light curves of a number of active galactic nuclei monitored since 1969 at the Rosemary Hill Observatory, in order to derive the time scales of their long-term baselevel fluctuations. In the case of the baseline meanderings of BL Lac, they found a time scale of 6.5-7.7 years, depending on the adopted method, with a best fit value of 7.2 years. A similar value was obtained by Hagen-Thorn et al. (1997), who inferred a periodicity of 7.8 years by using the method of whitening a time series on optical data from 1968 to 1989 . The Jurkevich method was used by Fan et al. (1998) to analyse a century of data: they concluded in favour of a long-term period of 14 years, and confirmed the short-term periods of 0.60 and 0.88 years already reported by Webb et al. (1988). More recently, Hagen-Thorn et al. (2002) have found evidence of a similar periodicity (308 days) in both the photometric and polarimetric optical behaviour in the period 1980-1991. They suggest that periodic components may be transient phenomena and that this may explain different results obtained by different authors.

Quasi-periodic variations have been found by Kelly et al. (2003) in the radio light curves of the University of Michigan Radio Astronomy Observatory (UMRAO); by means of a cross-wavelet transform algorithm they inferred a characteristic period of 1.4 years for the $4.8 \mathrm{GHz}$ flux variations, 3.7 years for the $8.0 \mathrm{GHz}$ ones, and 3.5, 1.6, 0.7 years for the $14.5 \mathrm{GHz}$ flux changes.

As for the possible correlation between the flux variations detected in the optical and radio bands, Tornikoski et al. (1994a) reported on a nearly simultaneous optical and radio outburst observed in spring 1993. Other works on this subject always found radio variations noticeably delayed with respect to the optical ones (Bregman et al. 1990; Hufnagel \& Bregman 1992; Tornikoski et al. 1994b; Clements et al. 1995; Hanski et al. 2002). On the contrary, the correlation between flux variations at different radio wavelengths has been found very strong, with no or short time delay.

Preliminary results of the present work were already published in Raiteri (2003), for both the periodicity and cross-correlation analyses.

This paper is organized as follows: in Sect. 2 the "historic" optical and radio light curves back to 1968 are presented. The periodicity analysis on both the optical and radio data is performed in Sect. 3, while Sect. 4 deals with cross-correlation analysis between optical and radio data and between radio data at different frequencies. Final considerations are outlined in Sect. 5.

\section{Historic optical and radio light curves}

As mentioned in the Introduction, four WEBT campaigns have been organized up to now on BL Lacertae. The data collected during these campaigns, and in particular during the longterm campaigns in 2000-2001 and 2001-2002, have greatly enhanced the amount of photometric data available to study the flux behaviour of this source. In Villata et al. (2004) UBVRI light curves of BL Lacertae from 1994 to 2002 have been presented and a detailed colour analysis has been performed. In the present paper we want to perform time series and cross-correlation analyses on both the optical and radio light curves and hence we need the longest data trains possible. Consequently, in order to obtain a well-sampled and time-extended optical light curve, a composite $R$-band light curve has been constructed: all $B$-band magnitudes before JD = 2449401 were converted into $R$-band magnitudes adopting the mean colour index $B-R=1.7$, and added to the true $R$-band data after that date. The result is a light curve extending back 

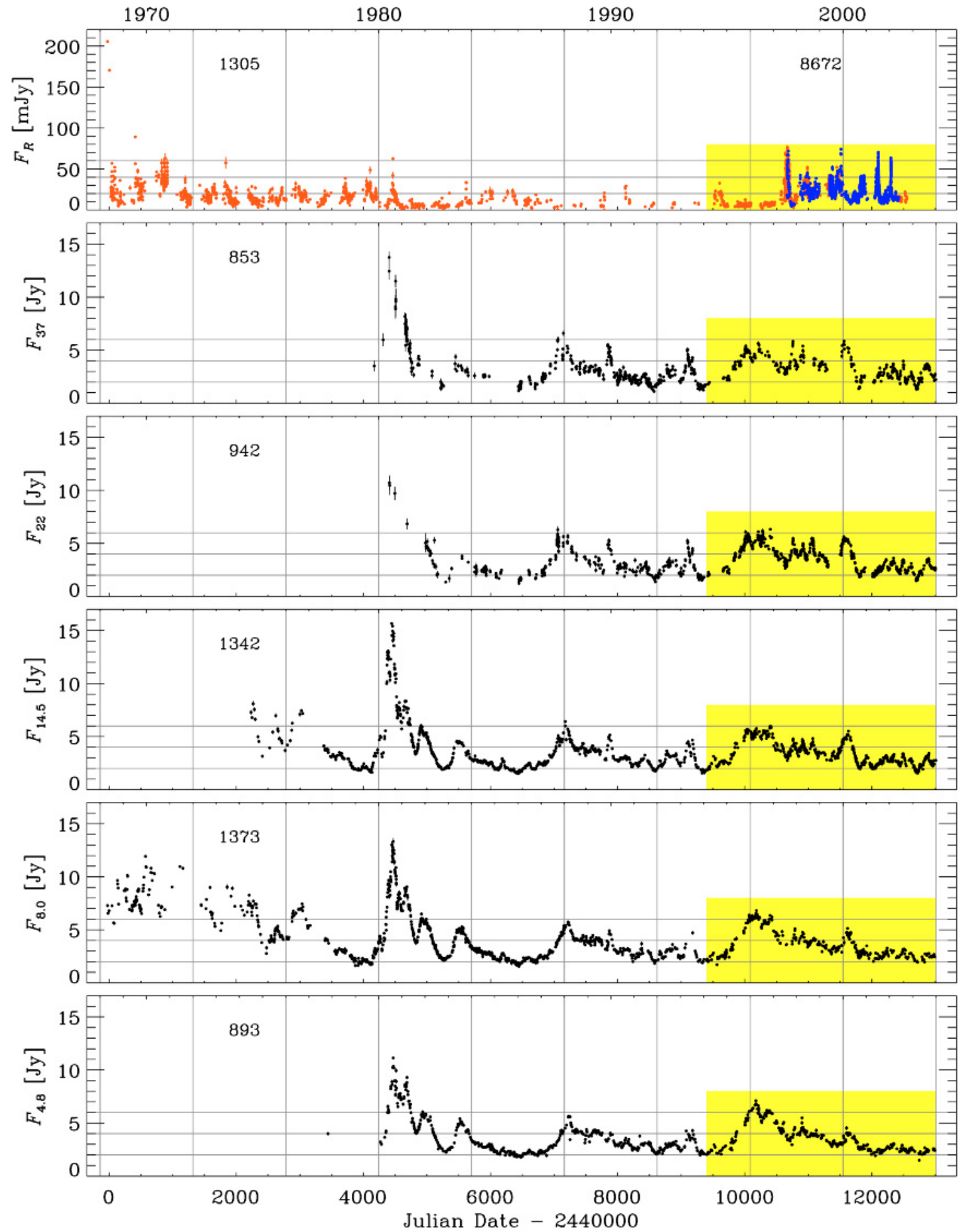

Fig. 1. Composite $R$-band flux light curve (top, mJy) and radio light curves (Jy) at 37 and $22 \mathrm{GHz}$ from the Metsähovi Radio Observatory and at 14.5, 8.0, and $4.8 \mathrm{GHz}$ from UMRAO; red (grey) optical dots represent literature data, while blue (dark) ones show data taken by the WEBT collaboration; grey horizontal and vertical lines have been drawn to guide the eye through the flux variations; the yellow (shaded) area highlights the period considered by Villata et al. (2004); the number of data is indicated in each panel: in the case of optical data, the $R$-converted $B$ data have been distinguished from the "true" $R$-band fluxes inside the yellow (shaded) region.

to 1968 , containing a total of 9977 data, of which 1305 (corresponding to 951 "nights") are $R$-converted $B$ magnitudes, and the remaining 8672 (1041 "nights") are original $R$ magnitudes ${ }^{1}$.

1 The "true" $R$-band data after JD $=2449401$ are from Villata et al. (2004), while the bulk of the $B$-band data before that date are from the collection by Fan \& Lin (2000) and a few ones from Maesano et al.(1997) and Bai et al. (1999). Some of the data
Magnitudes have been transformed into fluxes and the contribution of the host galaxy has been subtracted as described in Villata et al. (2004). The resulting composite, galaxysubtracted flux light curve is shown in the upper panel of Fig. 1.

collected by Fan \& Lin (2000) have been corrected after comparison with the original papers. 
Red (grey) dots represent literature data, while blue (dark) points are data collected by the WEBT. The yellow (shaded) area highlights the period analysed by Villata et al. (2004).

In the same figure radio light curves at 37 and $22 \mathrm{GHz}$ from the Metsähovi Radio Observatory and at 14.5, 8.0, and 4.8 GHz from the University of Michigan Radio Astronomy Observatory (UMRAO) are shown.

One can easily see that the radio behaviour at all wavelengths seems to follow a common path, while no kind of correlation with the optical trend is recognizable on the ground of a mere visual inspection.

Remarkable features of these light curves are the two extremely high optical fluxes detected in 1968, and the big radio outburst observed at all radio frequencies in 1980 .

The light curve at $8.0 \mathrm{GHz}$, which is the most extended in time, shows a base level that first decreases (until JD 2444000 ) and then remains almost constant. A similar trend can also be recognized in the less extended light curve at $14.5 \mathrm{GHz}$.

Moreover, one can notice that the radio base level seems to be lower at the higher frequencies. This is a feature that was already noticed in another BL Lac object, S5 0716+71 (Raiteri et al. 2003).

As for the radio outburst amplitude, the common behaviour of BL Lac objects is that the variation amplitude increases with increasing frequency. By looking at Fig. 1, where horizontal and vertical lines have been drawn to make the visual inspection easier, one can see that this is indeed the case for a number of radio events, such as the prominent outburst occurred in 1980 and the more modest events of 1987-1988, 1989-1990, 1993, and in the period 1997-2003. However, there are also events whose amplitude is higher at the lower frequencies, as in 1983 and 1996.

\section{Periodicity analysis}

As quoted in the Introduction, periodicities on different time scales have been found by a number of authors when analysing optical or radio light curves of BL Lacertae. Sometimes periodicities were recognized over a limited period of time, as if due to a transient periodic phenomenon.

In the following both the radio and optical light curves of BL Lac are analysed with the same statistical tools: the discrete Fourier transform (DFT) following the prescription of Press et al. (1992), the discrete autocorrelation function (ACF; Edelson \& Krolik 1988; Hufnagel \& Bregman 1992), and the structure function (SF; Simonetti et al. 1985).

All of them have their own merits and faults, but we think that, when used together, they can give a reliable insight into the source behaviour. We expect that, if a periodicity is present, it will show up as a clear peak in both the DFT and ACF and as a minimum in the SF (or a maximum in - SF), and, if the data train extension allows it, in the ACF and SF signatures will appear also at times which are multiples of the period. In particular, $n$ periodic events will produce $n-1$ signatures. For the sake of comparison, we present DFT, ACF, and -SF plots together in the same figure. Notice that the time scales derived from $\mathrm{ACF}$ and $\mathrm{SF}$ reported in the following do not refer to the peak of the distribution, but to its centroid, $\tau_{\mathrm{c}}=\sum_{i} \tau_{i} f_{i} / \sum_{i} f_{i}$, where $f_{i}$ is the value of the function in the $i$ th bin and the sum runs over all the points for which $f_{i}$ is greater than a certain percentage of the peak value, usually $80 \%$ (Peterson et al. 2001).

\subsection{Search for periodicities in the radio bands}

The results of the application of the above three methods to the radio fluxes at $4.8 \mathrm{GHz}$ are displayed in Fig. 2. In the upper panel the power spectrum of the DFT shows a prominent peak with significance well above 0.001 (blue dotted line), which suggests a period of 2984 days. Two main features are also found in both the ACF and -SF plots, indicating time scales of 2843 and 5718 days in the ACF, and of 2839 and 5689 days in the SF. When averaging all the above signals coming from the DFT, ACF, and SF, a "best-fit" period of 2874 days, i.e. 7.87 years, is derived.

In the middle panel of Fig. 2 the results of the Monte Carlo technique known as "flux redistribution/random subset selection" (FR/RSS; Peterson et al. 1998; see also Raiteri et al. 2003) are also shown. The red dotted line indicates the mean ACF obtained after autocorrelating 1000 Monte Carlo realizations. This mean ACF appears to match the "true" ACF fairly well, the only remarkable deviation showing up after $\tau \sim 6000$ days. Moreover, the two insets show the percentage of occurrence of a given centroid value in the 1000 Monte Carlo realizations, which allows to estimate the probability of the result. In this specific case, we can say that at the $93.5 \%$ confidence level the first time scale ranges from 2800 to 2880 days and that at the $81.1 \%$ level the second time scale is between 5680 and 5760 days. This certainly reconciles the results of the ACF with those of the SF, but the time scale of the Fourier analysis remains a bit longer.

The DFT also shows another major peak at 5787 days (similar to the ACF and SF long periods) and other, minor peaks at 1436,1117 , and 941 days (3.93, 3.06, and 2.58 years), which have however significance better than 0.001 . The 3.5 year period found by Kelly et al. (2003) lies just in the middle of the first two minor peaks. However, neither the ACF nor the SF support these shorter time scales.

The same analysis performed on the $8.0 \mathrm{GHz}$ fluxes in the same period of the $4.8 \mathrm{GHz}$ ones (JD > 2444000 ) leads to quite similar results, with an average period of 7.85 years. However, when considering the whole $8.0 \mathrm{GHz}$ light curve (since 1968), the results are strongly affected by the already mentioned decreasing trend of the base level in the first 10 years of the light curve. In particular, this trend, showing up as a typical "shot noise" shape in the ACF plot, overcomes the features we are looking for. Thus, before applying the three methods, we subtracted from the $8.0 \mathrm{GHz}$ fluxes a linear decreasing trend up to JD $=2444000$ and a constant value afterwards.

The result of the periodicity analysis on the "cleaned" $8.0 \mathrm{GHz}$ data is shown in Fig. 3; notice that the $\tau$ scale is more extended than in the $4.8 \mathrm{GHz}$ case, since the $8.0 \mathrm{GHz}$ light curve is more extended in time. In this figure one can recognize features similar to those found in the $4.8 \mathrm{GHz}$ data 

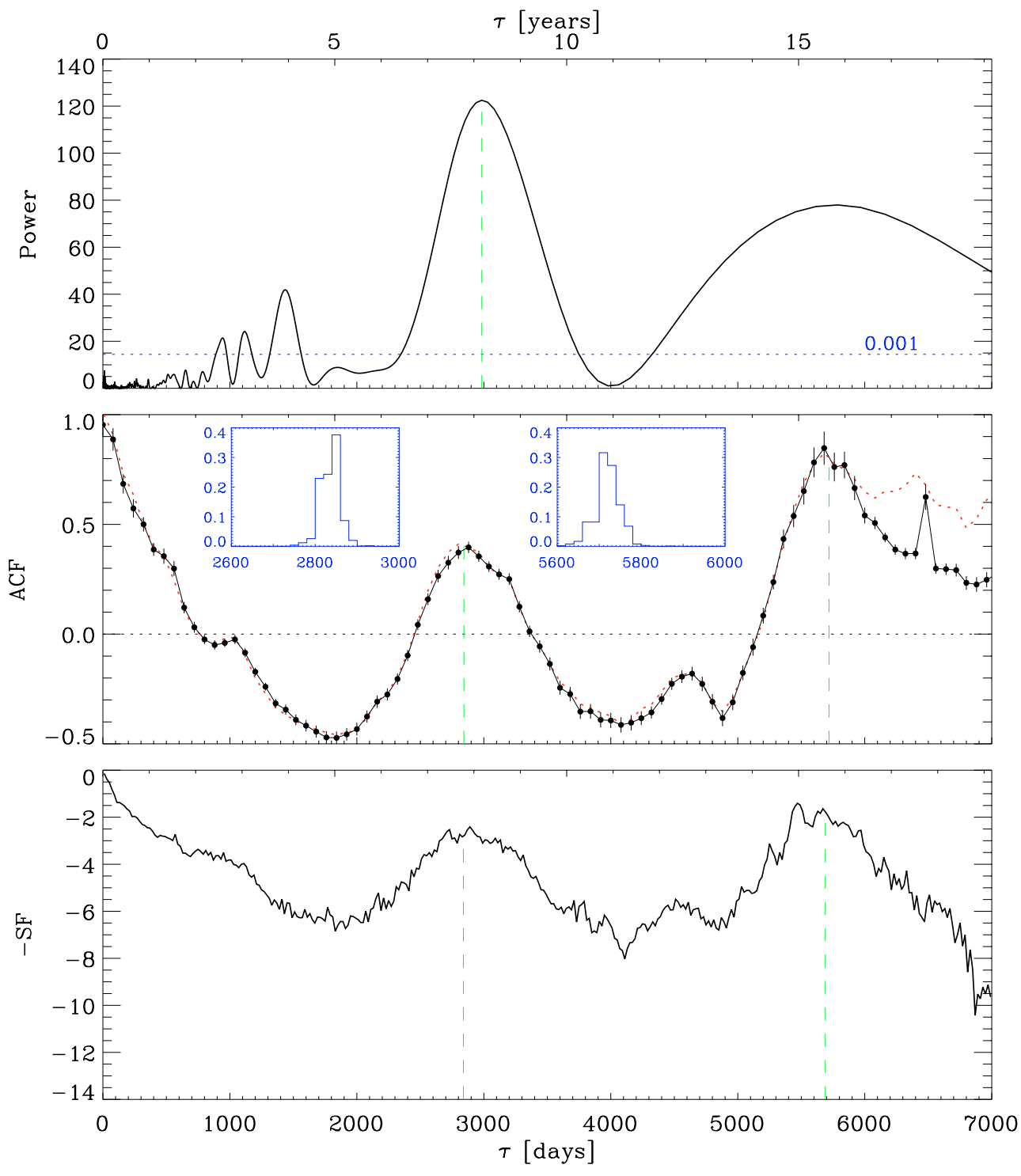

Fig. 2. Power spectrum of the discrete Fourier transform (top panel), autocorrelation function (middle panel), and structure function changed in sign (bottom panel) obtained from the $4.8 \mathrm{GHz}$ data; in the top panel the blue dotted line marks the level above which the significance is better than 0.001 ; dashed vertical lines indicate peak/centroid values of the major peaks. In the middle panel the mean ACF obtained after 1000 FR/RSS Monte Carlo realizations is shown (red dotted line), as well as the percentage of occurrence of the centroid values in the same realizations (insets). analysis. The most significant peak of the power spectrum (upper panel) implies a period of 2991 days. Centroid values of the ACF are 2720 and 5718 days; Monte Carlo FR/RSS calculations tell us that at $89.6 \%$ confidence level $\tau_{\mathrm{c}}$ for the first period is between 2660 and 2740 days, while that of the second period lies between 5660 and 5740 at a $91.6 \%$ confidence level (see insets in the middle panel of Fig. 3). An average of the DFT and ACF results gives a "best-fit" period of 7.82 years, in fair agreement with the previous findings notwithstanding the 10 year longer data train. Moreover, the ACF also shows a very broad maximum from about 23.5 to about 26 years, that is at about three times the period. In this sense, one can find here, in the most time extended radio light curve, a further hint in favour of a $\sim 8$ year periodicity. As for the SF (Fig. 3, lower panel), it appears quite noisy with rather broad peaks. However, if we plot coloured strips corresponding to the $\tau_{\mathrm{c}}$ ranges found through the Monte Carlo FR/RSS simulations of the ACF, and also report the location of the power spectrum peak (coloured line), we can see that these previous results are in fair agreement with the time scales that the SF analysis would suggest. Moreover, a prominent, broad maximum extends from 23.5 to 26 years, confirming the feature already noticed in the ACF plot.

Other peaks appear in the DFT plot at 5914 days (about twice the period) as well as at 1391 and 1162 days (3.81 and 3.18 years), to be compared with the 3.7 year period found by Kelly et al. (2003). It is interesting to notice that, unlike the $4.8 \mathrm{GHz}$ case, minor bumps are clearly visible around 3 years in both the ACF and -SF plots.

As in the case of the $8.0 \mathrm{GHz}$ data, also for the $14.5 \mathrm{GHz}$ ones we first cleaned the light curve for the linear decrease of the base level, which seems to occur up to JD $~ 2444000$. The results of the consequent periodicity analysis are shown in Fig. 4.

The major peak in the DFT power spectrum (upper panel) is found at 3035 days. The ACF shows two maxima with $\tau_{\mathrm{c}}=$ 2801 and 5803 days, which have confidence levels of $90.3 \%$ and $88.8 \%$ to lie in the ranges $2740-2820$ and 5740-5820 days, respectively. The average period derived from the $14.5 \mathrm{GHz}$ data is thus 7.97 years, a bit longer than in the previous cases. The SF is noisier; however, one can see that the results of both 

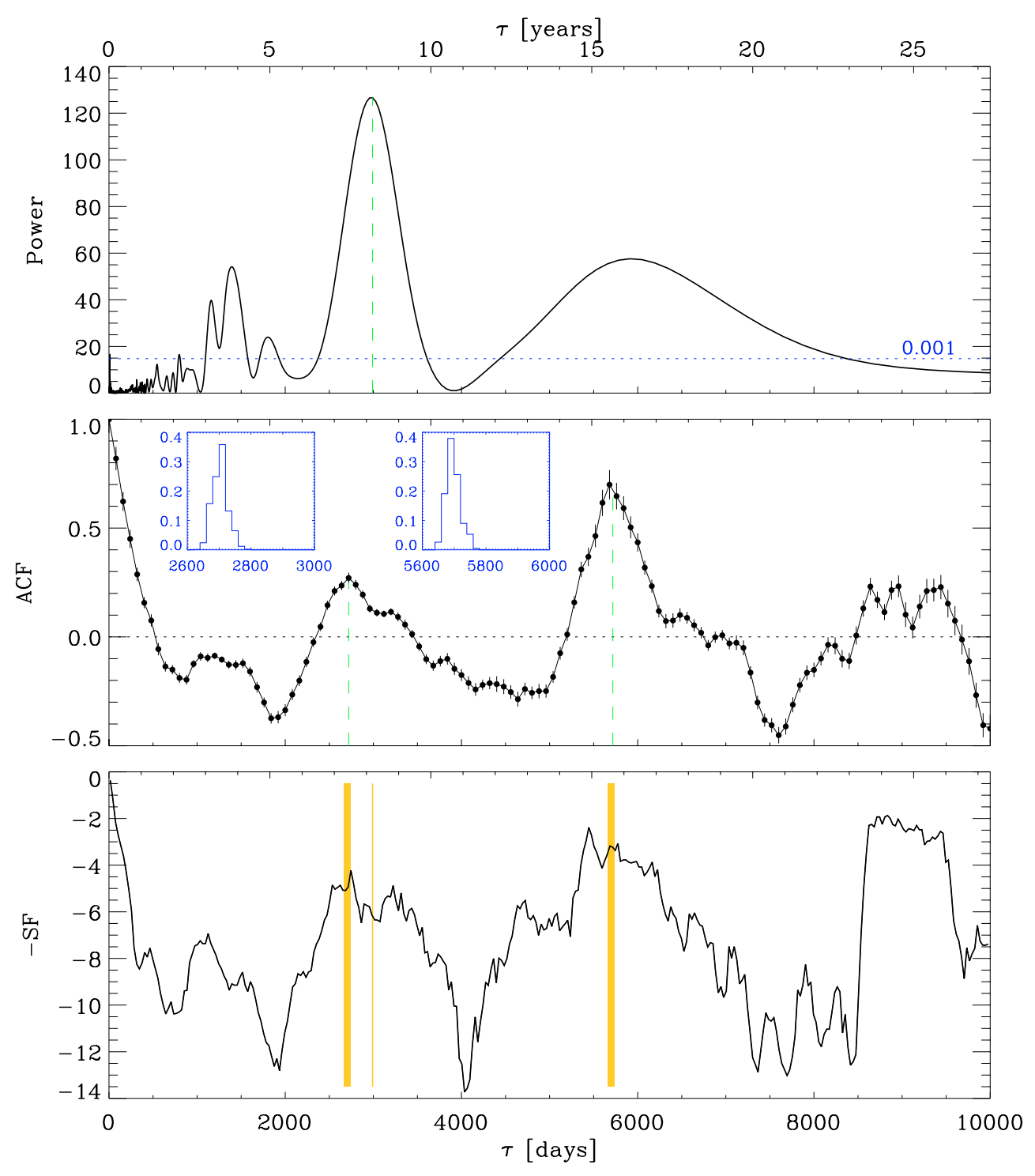

Fig. 3. Power spectrum of the discrete Fourier transform (top panel), autocorrelation function (middle panel), and structure function changed in sign (bottom panel) obtained from the $8.0 \mathrm{GHz}$ data; in the top panel the blue dotted line marks the level above which the significance is better than 0.001; dashed vertical lines in the first two panels indicate peak/centroid values of the major peaks. In the middle panel the percentage of occurrence of the ACF centroid values after $1000 \mathrm{FR} / \mathrm{RSS}$ Monte Carlo realizations is shown (insets). Coloured strips in the bottom panel indicate the centroid ranges obtained by the FR/RSS Monte Carlo simulations of the ACF, and the coloured line reports the position of the DFT peak.

the Monte Carlo FR/RSS method on the ACF (coloured strips in the bottom panel of Fig. 4) and the DFT (coloured line) are not in contradiction with the main features of the SF.

Also in the DFT plot of the $14.5 \mathrm{GHz}$ data other periodicities come out, with time scales similar to the previous cases:

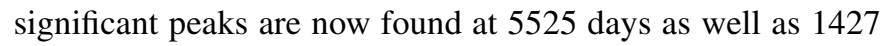
and 1146 days (3.91 and 3.14 years). The last two can be compared with the period of 3.5 years found by Kelly et al. (2003). Here again we can find some confirmation of the existence of a 3-4 year typical time scale in the bump showed more clearly by the -SF plot and less strongly by the ACF one.

The same investigation was performed on the 22 and $37 \mathrm{GHz}$ light curves, finding similar results, but with lower confidence due to the worse sampling.

In Table 1 we report the main numbers on the $\sim 8$ year radio periodicity found in this section.

In conclusion, our analysis on the radio light curves of BL Lacertae suggests a periodic behaviour with a time scale of $\sim 8$ years. By looking at Fig. 1, one can see that this period corresponds to the time separation between the outbursts occurred in 1980, 1987-1988, and 1995-1996. The limited time extension of our light curves does not allow us to emphasize this result too much, but it goes without saying that the detection of a major radio outburst in 2004 would strengthen it.

The shorter (about half) time scale of 3-4 years found in our analysis is determined by the presence of the outbursts occurred in 1976, 1983, and 1999-2000. This signal is less clear due to the lack of an analogous event around 1991-1992.

\subsection{Search for periodicities in the optical band}

We have just stated that $\mathrm{a} \sim 8$ year periodicity seems to characterize the radio light curves of BL Lacertae; this periodicity is surprisingly in agreement with the $7.8 \pm 0.2$ year period found by Hagen-Thorn et al. (1997) in the optical band, and to the values of periodicity of the optical base level identified by Smith \& Nair (1995).

We now want to see whether the time series analysis on the composite optical light curve of BL Lac that we have reconstructed (see Sect. 2) can confirm these previous findings.

Figure 5 shows the results of our analysis on the optical magnitudes: while both ACF and SF are extremely noisy 

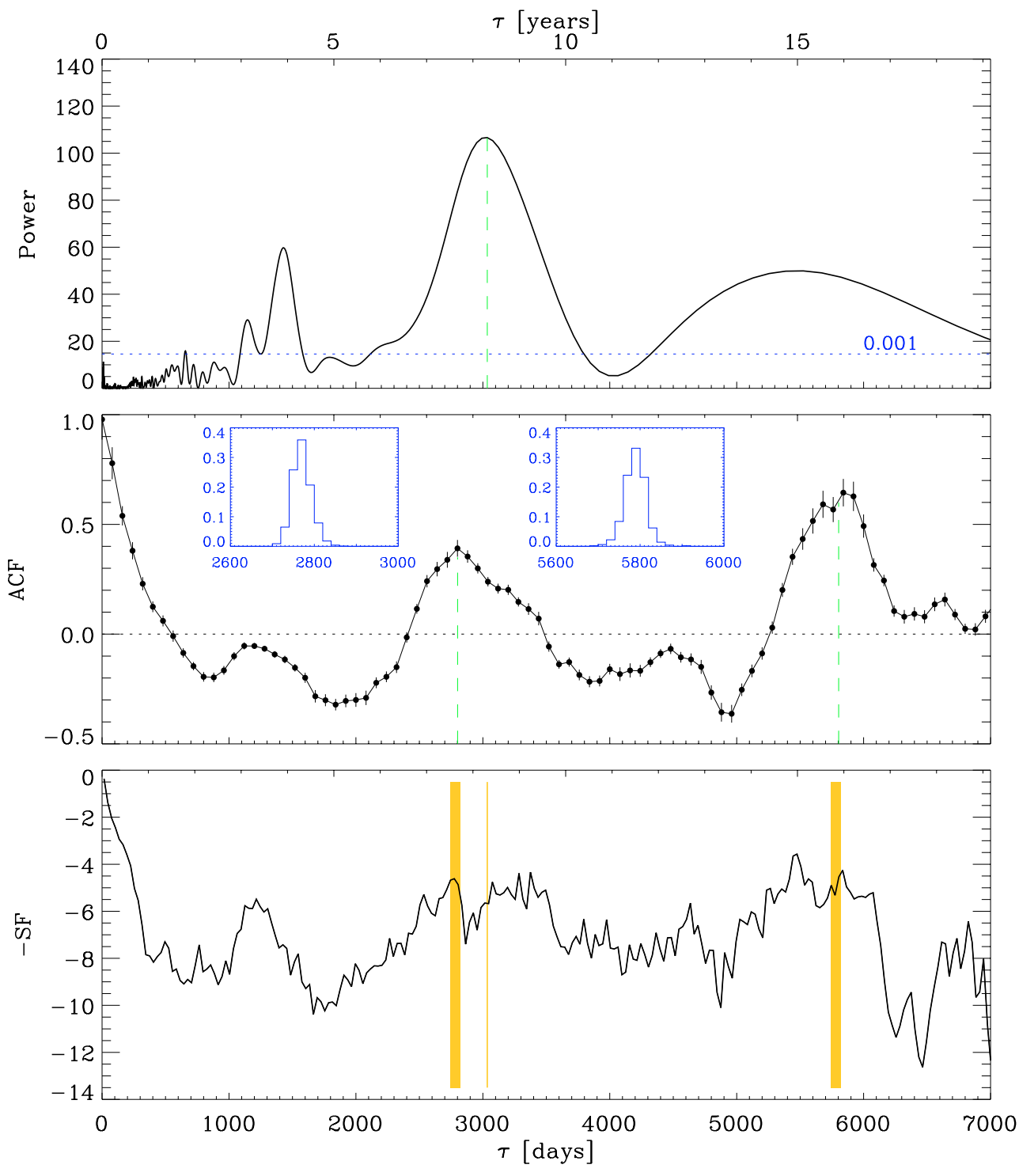

Fig. 4. Power spectrum of the discrete Fourier transform (top panel), autocorrelation function (middle panel), and structure function changed in sign (bottom panel) obtained from the $14.5 \mathrm{GHz}$ data; in the top panel the blue dotted line marks the level above which the significance is better than 0.001 ; dashed vertical lines in the first two panels indicate peak/centroid values of the major peaks. In the middle panel the percentage of occurrence of the ACF centroid values after 1000 FR/RSS Monte Carlo realizations is shown (insets). Coloured strips in the bottom panel indicate the centroid ranges obtained by the FR/RSS Monte Carlo simulations of the ACF, and the coloured line reports the position of the DFT peak.

Table 1. Comparison among the results found at different frequencies with different methods on the main radio periodicity of $\sim 8$ years.

\begin{tabular}{lccccc}
\hline \hline Method & $4.8 \mathrm{GHz}$ & $8.0 \mathrm{GHz}$ & $14.5 \mathrm{GHz}$ & $22 \mathrm{GHz}$ & $37 \mathrm{GHz}$ \\
\hline DFT (days) & 2984 & 2991 & 3035 & 3180 & 3154 \\
ACF (days) & $2843,5718 / 2$ & $2720,5718 / 2$ & $2801,5803 / 2$ & $2968,5924 / 2$ & $2799,5760 / 2$ \\
SF (days) & $2839,5689 / 2$ & & & & \\
\hline Mean (years) & 7.87 & 7.82 & 7.97 & 8.31 & 8.06 \\
\hline
\end{tabular}

and do not reveal any characteristic time scale, the DFT (solid line) shows significant peaks at 7.25, 10.28, 14.65, 4.78, and 1.2 years. The red dotted line in the upper panel of the figure displays the power spectrum obtained by running the DFT on the optical fluxes: the results previously obtained on the magnitudes are substantially confirmed, but peaks have now a lower significance. In particular, the peak at about 7 years appears strongly reduced with respect to that at $\sim 10$ years. On the contrary, no significant changes appear in the ACF and -SF plots. By limiting the Fourier analysis to the period considered by Hagen-Thorn et al. (1997), i.e. up to 1989, we also find the 7.8 year signal, thus confirming their result even if the dataset is not the same. However, since this period is not confirmed by the other two methods, the existence of a periodicity in the optical light curve of BL Lacertae must be regarded with caution.

\section{Cross-correlation analysis}

In this section the discrete correlation function (DCF) method is applied to the radio and optical light curves in order to look for correlated variations and possible time lags.

\subsection{Cross-correlation between radio light curves}

We have already noticed in Sect. 2 that the behaviour of the radio light curves of BL Lac at different wavelengths is very 

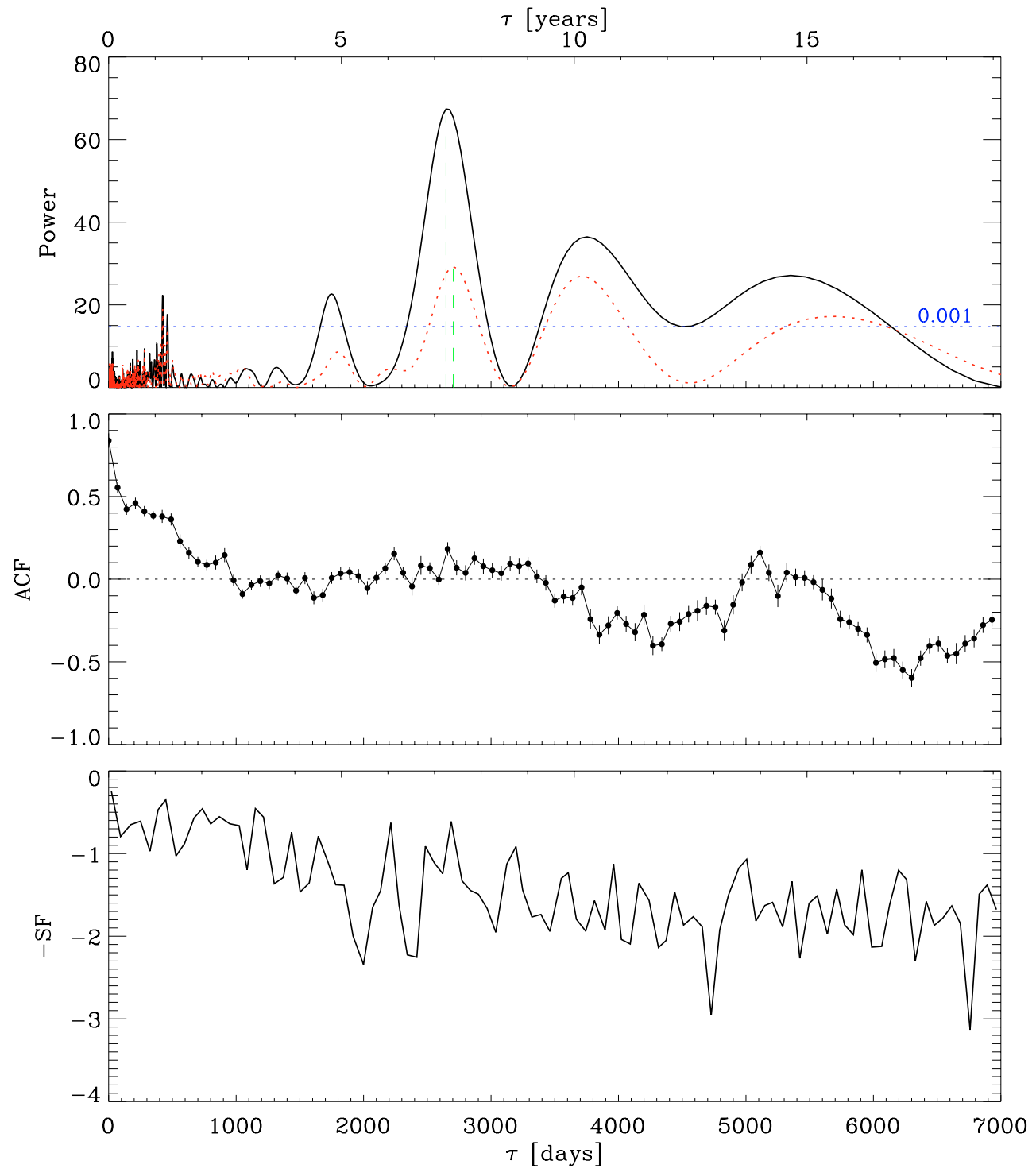

Fig. 5. Power spectrum of the discrete Fourier transform (top panel), autocorrelation function (middle panel), and structure function changed in sign (bottom panel) obtained from the optical magnitudes; in the top panel the red dotted curve displays the power spectrum obtained from the optical fluxes, and the blue dotted line marks the level above which the significance is better than 0.001 . similar, as expected. A radio event in one band always has a counterpart in the other radio bands, even if the variation amplitude can either increase or decrease with frequency.

The cross-correlation analysis run on the radio light curves of a number of other BL Lac objects has shown how variations at lower frequencies may lag those at the higher frequencies, the time delay increasing with the frequency separation.

In the following we cross-correlate the radio light curves shown in Fig. 1 starting from JD = 2446500 in order to consider the best sampled period only and, at the same time, to avoid the major imprint of the 1980 outburst, which in addition is not well sampled in all bands.

Figure 6 presents the results of the DCF between the $22 \mathrm{GHz}$ fluxes and the UMRAO data at $14.5,8.0$, and $4.8 \mathrm{GHz}$. The correlation is quite good, since the DCF peak value exceeds 0.9 in all the three cases. The calculation of the distribution centroid confirms that a delay is actually occurring: variations at $14.5,8.0$, and $4.8 \mathrm{GHz}$ are observed after those seen at $22 \mathrm{GHz}$ with an average lag of 19, 39, and 78 days, respectively.
Other cases are reported in Fig. 7; again the correlation is strong, and there is evidence of delays. Indeed, by calculating centroids one can estimate that changes in the $14.5 \mathrm{GHz}$ light curve precede those at $8.0 \mathrm{GHz}$ by 19 days and those at $4.8 \mathrm{GHz}$ by 59 days, while variations at $4.8 \mathrm{GHz}$ lag those at $8.0 \mathrm{GHz}$ by 40 days.

Notice that the above picture is self-consistent, since, for instance: $\tau_{\mathrm{c}}(14.5,8.0)+\tau_{\mathrm{c}}(8.0,4.8)=\tau_{\mathrm{c}}(14.5,4.8)$, and $\tau_{\mathrm{c}}(22,14.5)+\tau_{\mathrm{c}}(14.5,4.8)=\tau_{\mathrm{c}}(22,4.8)$.

\subsection{Cross-correlation between optical and radio light curves}

As discussed in the Introduction, several authors investigated the possibility that correlated variations in the optical and radio bands do exist.

Our analysis by means of the DCF has not revealed any hint of correlation between the optical and radio total light curves.

However, if we consider the last, best-sampled period (1994-2003) highlighted in Fig. 1 and better displayed 


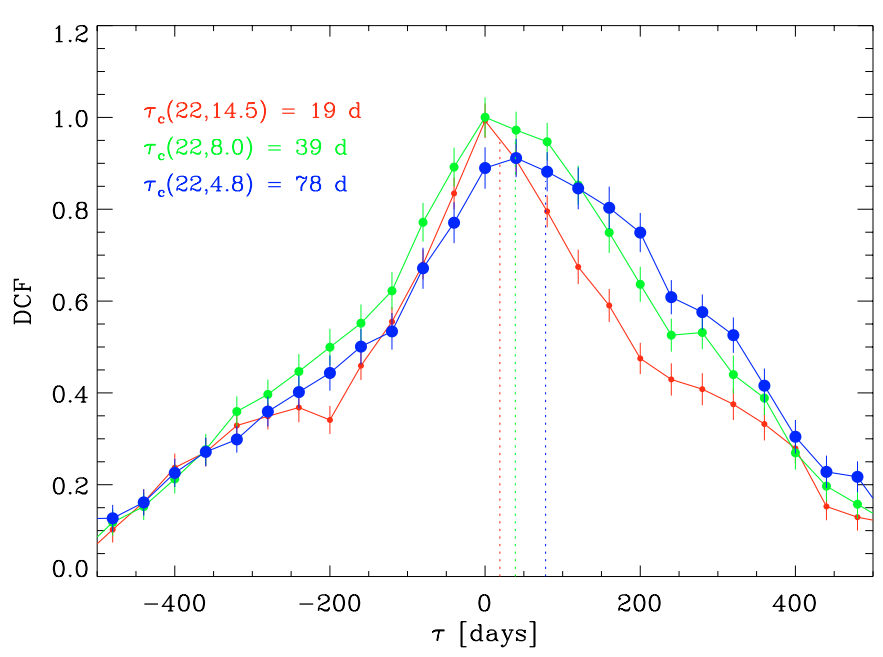

Fig. 6. Discrete correlation function between 22 and $14.5 \mathrm{GHz}$ data (red/grey), 22 and $8.0 \mathrm{GHz}$ data (green/light grey), and 22 and $4.8 \mathrm{GHz}$ data (blue/dark); vertical dotted lines indicate the distribution centroids.

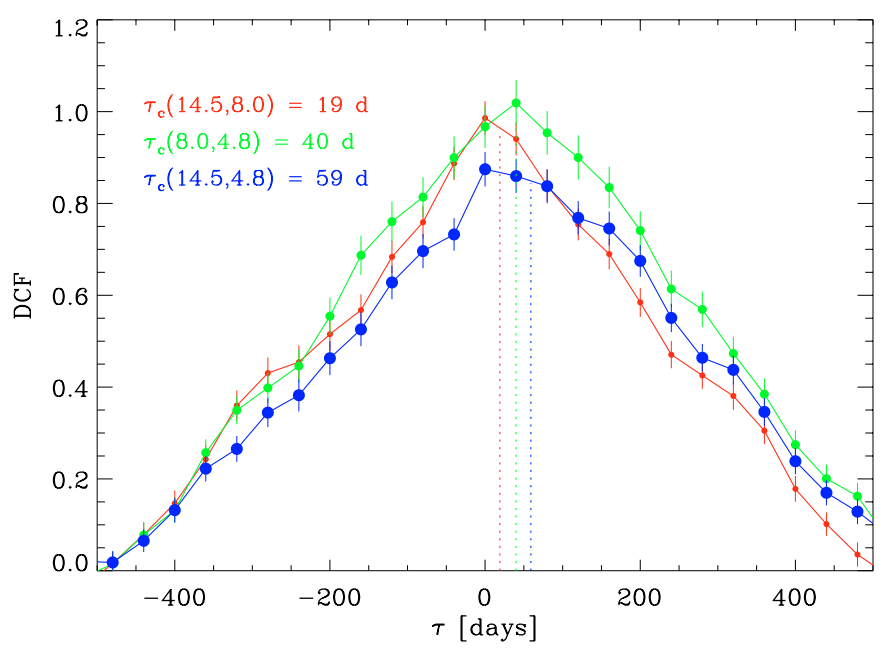

Fig. 7. Discrete correlation function between 14.5 and $8.0 \mathrm{GHz}$ data (red/grey), 8.0 and $4.8 \mathrm{GHz}$ data (green/light grey), and 14.5 and 4.8 GHz data (blue/dark); vertical dotted lines indicate the distribution centroids.

in Fig. 8, a correlation signal does appear, which is stronger for the higher radio frequencies, indicating a radio delay of about 100 days.

As we have already noticed, the radio light curves seem to be the combination of a harder and a softer component, the softer component providing the bulk of the radio emission and the harder one almost disappearing at the lowest frequency. A similar behaviour was already noticed in the BL Lac object S5 0716+71 (see Raiteri et al. 2003), with the main difference that in that case the softer component appeared to be almost constant, essentially a base level. On the contrary, in BL Lac the soft component varies a lot.

The above result that the optical-radio correlation is more evident at the higher radio frequencies (where the harder component is stronger) suggests that it is the harder component which correlates with the optical fluxes, as one can expect.
In other words, we are envisaging a picture where the mechanisms causing the optical events also affect the flux behaviour at lower frequencies, fading away at the longer radio wavelengths. Thus, the soft component would act as a confusing element and we try to remove it in the following way.

In Fig. 8 the top panel shows the optical fluxes (both WEBT and literature data), while the following three panels display the radio fluxes at $22,14.5$, and $4.8 \mathrm{GHz}^{2}$. The first step consists in approximating the radio light curves by a smoothing cubic spline, after binning the data over 30 days. The result is plotted together with the light curves themselves. In the last two panels the splines at 22 (blue/dark) and $14.5 \mathrm{GHz}$ (green/light grey) are compared with the $4.8 \mathrm{GHz}$ one (red/grey). The regions where the higher-frequency spline exceeds the lowerfrequency one are highlighted (yellow/shaded areas) to distinguish the hard-spectrum events, which are expected to have optical counterparts.

A way for isolating the hard component is to calculate the spline hardness ratios $H_{22}=F_{22} / F_{4.8}$ and $H_{14.5}=F_{14.5} / F_{4.8}$. As expected, the cross-correlation of these two quantities with the optical fluxes is much better than when considering the original radio fluxes: the hard radio variations seem to lag the optical ones by about 100 days (see Fig. 9). In the top panel of Fig. 8 the hardness ratios $H_{22}$ (blue/dark line) and $H_{14.5}$ (green/light grey line) are overplotted on the optical data after shifting them by -100 days (as well as raising them to the fourth power and multiplying by 10 for graphic convenience). As one can see, almost all the major optical events find their radio counterpart. It is also interesting to notice that the exceptions represented by the two optical outbursts in 2000 and 2002 have been recognized by Villata et al. (2004) as bluer-thanusual events (see Figs. 5 and 9 therein). Indeed, a harder optical spectrum likely implies a lower radio flux.

\section{Conclusions}

In this paper we have analysed the historical optical and radio (from 4.8 to $37 \mathrm{GHz}$ ) light curves of BL Lacertae from 1968 to 2003. Most of the optical data comes from the paper by Villata et al. (2004), who assembled literature data together with data taken by the members of the Whole Earth Blazar Telescope (WEBT) collaboration in the period 1994-2002, especially during the WEBT campaigns of 1999, 2000-2001, and 2001-2002. Radio light curves are the results of the monitoring programs running at the University of Michigan and Metsähovi radio observatories.

A detailed periodicity analysis on the radio data from 4.8 to $37 \mathrm{GHz}$ reveals a clear evidence of a $\sim 8$ year period, which is supported by the results from three different methods: discrete Fourier transform, autocorrelation function, and structure function. A less-clear periodicity is also found in the optical light curve, with a similar time scale: $7-8$ years.

The flux variations in the radio bands at different frequencies appear to be well correlated, with gradual time delays

\footnotetext{
2 The $37 \mathrm{GHz}$ light curve has not been considered in this analysis since in this period it is undersampled of a factor 2 with respect to the close $22 \mathrm{GHz}$ one and a couple of important radio events are missing.
} 

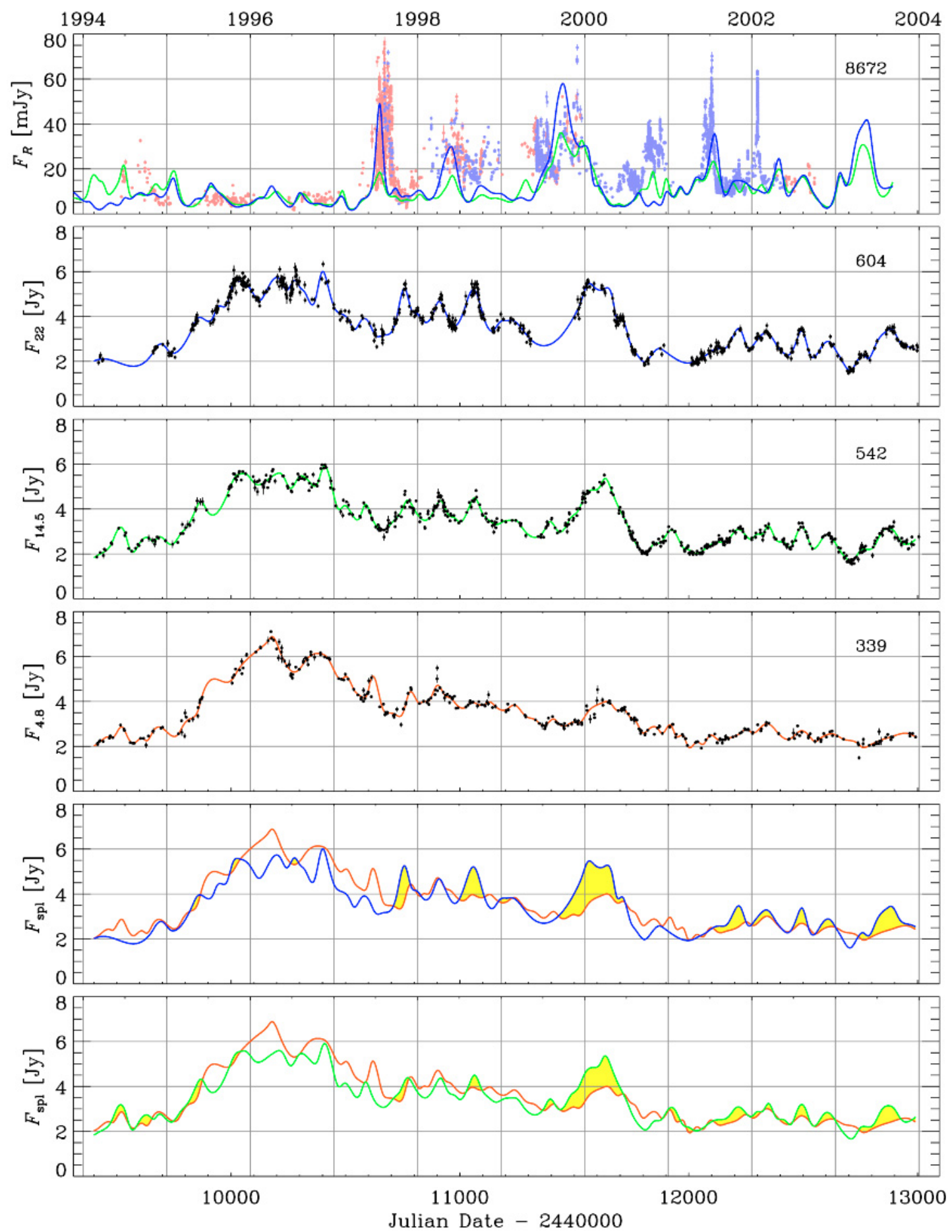

Fig. 8. $R$-band flux light curve (top, mJy) and radio light curves (Jy) at 22, 14.5, and $4.8 \mathrm{GHz}$ with their smoothing cubic splines underplotted; grey horizontal and vertical lines have been drawn to guide the eye through the flux variations; the fifth and sixth panels show the 22 (blue/dark) and $14.5 \mathrm{GHz}$ (green/light grey) splines, respectively, both compared with the $4.8 \mathrm{GHz}$ one (red/grey); the regions where the higher-frequency spline exceeds the lower-energy one have been highlighted; in the top panel the corresponding hardness ratios have been plotted (after shifting, see text for details) for comparison with the optical fluxes.

(higher frequencies leading), from a few weeks between contiguous bands up to a few months when considering the largest frequency separations.

Radio light curves appear to be the combination of two components with different behaviour: while the bulk of the radio emission presents a softer spectrum, several events appear to be enhanced at the higher frequencies, suggesting the possibility of correlation with optical events. Indeed, crosscorrelation analysis in the best-sampled period 1994-2003 between the optical light curve and radio hardness ratios reveals that hard-spectrum radio events likely lag optical outbursts by more than 3 months. 


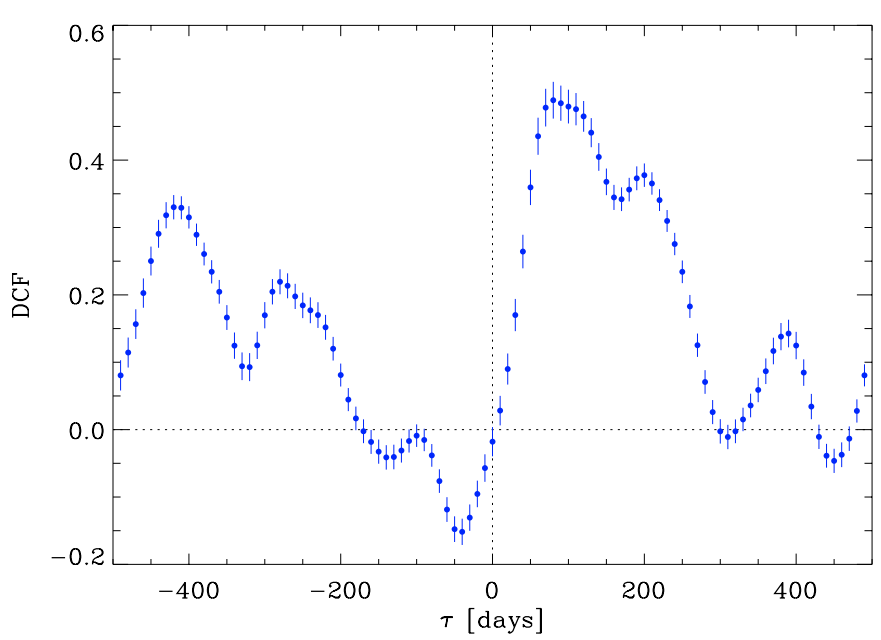

Fig. 9. Discrete correlation function between the optical fluxes and the hardness ratio $H_{22}$ (see text); a fair correlation is found at a time lag of $\sim 100$ days.

In conclusion, our analysis supports a scenario where the mechanisms giving rise to optical outbursts also affect the lower-energy flux behaviour, implying delayed radio events that become less evident with decreasing frequency. On the other hand, radio light curves are dominated by a softerspectrum component, possibly periodic, whose strong variations have not optical counterparts.

The delays between optical and radio variations and between flux changes at different radio frequencies as well imply that, whatever the variability mechanism is (e.g. shocks, magnetic reconnection, Doppler factor variations, etc.), the phenomenon propagates towards less and less opaque regions, with the harder-spectrum radio events probably occurring in a more compact region (closer to the optically emitting one) with respect to the site where the softer radio emission is produced.

We finally notice that the $\sim 8$ year periodicity of radio outbursts seems to gradually lengthen. Indeed, by looking at Fig. 1, one can see that the time separation between the 1980 and 1987-1988 outbursts is sensibly less than 8 years, while the following period (up to the 1995-1996 outburst) is a bit longer. Moreover, the absence of a brightening trend at the end of 2003 may indicate that the expected next outburst will be further delayed.

Acknowledgements. This research has made use of data from the University of Michigan Radio Astronomy Observatory, which is supported by the National Science Foundation and by funds from the University of Michigan. This work was partly supported by the European Community's Human Potential Programme under contract HPRN-CT-2002-00321, by the Italian Space Agency (ASI) under contract CNR-ASI $1 / \mathrm{R} / 27 / 02$, and by the Italian Ministry for University and Research (MURST) under grant Cofin 2001/028773. St.-Petersburg group was supported by Russian Federal Programs "Astronomy" (grant N 40.022.1.1.1001) and "Integration" (grant N B0029).

\section{References}

Bai, J. M., Xie, G. Z., Li, K. H., Zhang, X., \& Liu, W. W. 1999, A\&AS, 136,455

Böttcher, M., Marscher, A. P., Ravasio, M., et al. 2003, ApJ, 596, 847

Bregman, J. N., Glassgold, A. E., Huggings, P. J., et al. 1990, ApJ, 352,574

Carini, M. T., Miller, H. R., Noble, J. C., \& Goodrich, B. D. 1992, AJ, 104,15

Clements, S. D., Smith, A. G., Aller, H. D., \& Aller, M. F. 1995, AJ, 110,529

Edelson, R. A., \& Krolik, J. H. 1988, ApJ, 333, 646

Fan, J. H., \& Lin, R. G. 2000, ApJ, 537, 101

Fan, J. H., Xie, G. Z., Pecontal, E., Pecontal, A., \& Copin, Y. 1998, ApJ, 507, 173

Hagen-Thorn, V. A., Marchenko, S. G., Mikolaichuk, O. V., \& Yakovleva, V. A. 1997, Astron. Rep., 41, 154

Hagen-Thorn, V. A., Larionov, V. M., Jorstad, S. G., \& Larionova, E. G. 2002, AJ, 124, 3031

Hanski, M. T., Takalo, L. O., \& Valtaoja, E. 2002, A\&A, 394, 17

Hufnagel, B. R., \& Bregman, J. N. 1992, ApJ, 386, 473

Kelly, B. C., Hughes, P. A., Aller, H. D., \& Aller, M. F. 2003, ApJ, 591,695

Maesano, M., Montagni, F., Massaro, E., \& Nesci, R. 1997, A\&AS, 122,267

Ostorero, L., Villata, M., \& Raiteri, C. M. 2004, A\&A, 419, 913

Peterson, B. M. 2001, in The Starburst-AGN Connection 2001, (Singapore: World Scientific), 3

Peterson, B. M., Wanders, I., Horne, K., et al. 1998, PASP, 110, 660

Press, W. H., Teukolsky, S. A., Vetterling, W. T., \& Flannery, B. P. 1992, Numerical recipes in Fortran - The Art of Scientific Computing (Cambridge: Cambridge University Press)

Raiteri, C. M. 2003, in Proc. First ENIGMA Meet., ed. M. Hauser, U. Bach, \& S. Britzen, Max-Planck-Institut für Radioastronomie, Bonn

(http://www.lsw.uni-heidelberg.de/users/swagner/ Efiles/proceedingsEM1.pdf), 131

Raiteri, C. M., Villata, M., Aller, H. D., et al. 2001, A\&A, 377, 396

Raiteri, C. M., Villata, M., Tosti, G., et al. 2003, A\&A, 402, 151

Ravasio, M., Tagliaferri, G., Ghisellini, G., et al. 2002, A\&A, 383, 763

Sillanpää, A., Takalo, L. O., Pursimo, T., et al. 1996, A\&A, 315, L13

Simonetti, J. H., Cordes, J. M., \& Heeschen, D. S. 1985, ApJ, 296, 46

Smith, A. G., \& Nair, A. D. 1995, PASP, 107, 863

Tornikoski, M., Valtaoja, E., Teräsranta, H., \& Okyudo, M. 1994a, A\&A, 286, 80

Tornikoski, M., Valtaoja, E., Teräsranta, H., et al. 1994b, A\&A, 289, 673

Villata, M., \& Raiteri, C. M. 1999, A\&A, 347, 30

Villata, M., Raiteri, C. M., Sillanpää, A., \& Takalo, L. O. 1998, MNRAS, 293, L13

Villata, M., Mattox, J. R., Massaro, E., et al. 2000, A\&A, 363, 108

Villata, M., Raiteri, C. M., Kurtanidze, O. M., et al. 2002, A\&A, 390, 407

Villata, M., Raiteri, C. M., Kurtanidze, O. M., et al. 2004, A\&A, 421, 103

Webb, J. R., Smith, A. G., Leacock, R. J., et al. 1988, AJ, 95, 374 